\title{
AN ASYMPTOTIC PROPERTY OF GAUSSIAN PROCESSES. I $\left({ }^{1}\right)$
}

\author{
BY \\ HISAO WATANABE
}

1. Introduction. The study of the asymptotic properties of stochastic processes has a long history. Such researches were developed from those of the asymptotic behavior of the first $n$ of a sequence of independent random variables, namely, the so-called law of the iterated logarithm. A. Kolmogoroff proposed the final form of the very theory which is stated, without proof, in P. Lévy's book [4]. W. Feller [3] gave its complete proof. The final result on the Brownian motion corresponding to Feller's for the case of the partial sums of independent random variables were led by T. Sirao and T. Nisida [9]. Their result is stated below. We introduce the following notation. Let $M_{a}^{+}=\{\phi ; \phi$ is a positive, nondecreasing, real function on $[a, \infty)\}$. Let $\{B(t) ; 0<t<\infty\}$ a Brownian motion on a probability measure space $(\Omega, B, P)$. We put $v(t)=E\left(E\left(B(t)^{2}\right)\right)^{1 / 2}=t^{1 / 2}$.

\section{TheOrem A.}

$P\left(\right.$ there is a $t_{0}(\omega)$ such that $|B(t)| \leqq v(t) \phi(t)$ for all $\left.t>t_{0}(\omega)\right)=1$ or 0, according as, for some $a>0$,

$$
\int_{a}^{\infty}(1 / t) \phi(t) \exp \left(-\frac{1}{2} \phi^{2}(t)\right) d t, \quad \phi \in M_{a}^{+},
$$

converges or diverges, respectively.

It will be possible to generalize in several ways Theorem A which is true for the Brownian motion. In this paper, by use of the method of T. Sirao [8], we will give some results on a generalization of Theorem A.

2. Results. Let $\{x(t),-\infty<t<\infty\}$ be a real, separable, measurable Gaussian process defined on a probability measure space $(\Omega, \mathscr{B}, P)$. Without loss of generality, we may assume that $E(x(t))=0$. We put $r(t, s)=E(x(t) x(s))$ and $E\left(x^{2}(t)\right)=v^{2}(t)$. In the following, we will assume that $r(t, s)$ is continuous with respect to $t$ and $s$ and $v(t)$ is positive. And we put $\rho(t, s)=r(t, s) /(v(t) v(s))$.

In the following, we.will obtain some results on the asymptotic behavior of the process $x(t)$ as $t$ tends to infinity. To state the results, we introduce the following conditions.

Received by the editors April 1, 1969.

(1) Some results of this paper were previously announced in H. Watanabe [11], and an error found later in them which is corrected in this paper.

Copyright () 1970, American Mathematical Society 
(A.1) There are positive constants $\delta_{1}, C_{1}$ and $T_{1}$ such that there holds

$$
\rho(t, t+h) \geqq 1-C_{1} h^{\alpha},
$$

for all $h$ in $\left(0, \delta_{1}\right)$ and all $t>T_{1}$ for some $\alpha$ with $0<\alpha \leqq 2$.

(A.1') There are positive constants $C_{2}, T_{2}$ and $\eta(0<\eta<1)$ such that

$$
\rho(t, t+h) \leqq \max \left(1-C_{2} h^{\alpha}, \eta\right)
$$

for all $t>T_{2}$ for some $\alpha$ with $0<\alpha \leqq 2$.

(A.2) The limit

$$
\lim _{s \rightarrow \infty} \rho(t, t+s) \cdot s=0,
$$

converges uniformly with respect to $t$.

THEOREM 1. Suppose that condition (A.1) is satisfied. If $\phi(t) \in M_{a}^{+}$and

$$
\int_{a}^{\infty} \phi(t)^{2 / \alpha-1} \exp \left(-\frac{1}{2} \phi^{2}(t)\right) d t<\infty
$$

for some $a>0$, then we have

$$
P\left(\exists t_{0}(\omega), x(t) \leqq v(t) \phi(t) \text { for all } t \geqq t_{0}(\omega)\right)=1 .
$$

THEOREM 2. Suppose that condition (A. $\left.1^{\prime}\right)$ and (A.2) are satisfied. If $\phi(t) \in M_{a}^{+}$ and

$$
\int_{a}^{\infty} \phi(t)^{2 / \alpha-1} \exp \left(-\frac{1}{2} \phi^{2}(t)\right) d t=\infty
$$

for some $a>0$, then we have

$$
P(x(t)>v(t) \phi(t) \text { i.o. })=1,
$$

where i.o. means infinitely often.

Combining Theorems 1 and 2, we can easily show the following theorem.

TheOREM 3. Assume that conditions (A.1), (A.1') and (A.2) are satisfied at the same time for some $\alpha$. Let $\phi(t) \in M_{a}^{+}$for some a. Then

$$
P\left(\exists t_{0}(\omega), x(t) \leqq v(t) \phi(t) \text { for all } t \geqq t_{0}(\omega)\right)=1 \text { or } 0,
$$

according as the integral,

$$
\int_{a}^{\infty} \phi(t)^{2 / \alpha-1} \exp \left(-\frac{1}{2} \phi^{2}(t)\right) d t
$$

converges or diverges.

COROLlaRY 1. Under the same conditions in Theorem 3, we have, for every $\varepsilon>0$, $P\left(\exists t_{0}(\omega), x(t) \leqq v(t)(2 \log t+(2 / \alpha+1+\varepsilon) \log \log t)^{1 / 2}\right.$ for all $\left.t>t_{0}(\omega)\right)=1$. Moreover, we have for any $\varepsilon \geqq 0$,

$$
P\left(x(t)>v(t)(2 \log t+(2 / \alpha+1-\varepsilon) \log \log t)^{1 / 2} i . o .\right)=1 .
$$


From Corollary 1, assuming condition (A.1), it follows that, for every $\varepsilon>0$,

$$
\begin{array}{r}
P\left(\exists t_{0}(\omega), x(t)<v(t)\left((2 \log t)^{1 / 2}+(1 / \alpha+1 / 2+\varepsilon) \log \log t /(2 \log t)^{1 / 2}\right)\right. \\
\text { for all } \left.t>t_{0}(\omega)\right)=1 .
\end{array}
$$

Under the condition (A.1), the path functions $x(t)$ are almost certainly everywhere continuous. Then, for every fixed $t>0$, the quantity

$$
\eta(t)=\max _{0 \leqq u \leqq t} \frac{x(u)}{v(u)}
$$

will have a definite meaning. Given any positive, monotone, nondecreasing continuous function $\phi(t)$ for large $t$, there is a $t_{0}(\omega)$ such that $x(t)<v(t) \phi(t)$ for all $t \geqq t_{0}$, if and only if there is a $t_{0}(\omega)$ such that $\eta(t) \leqq \phi(t)$ for all $t \geqq t_{0}$. Hence, assuming condition (A.1), for every $\varepsilon>0$,

$$
\begin{array}{r}
P\left(\exists t_{0}(\omega), \eta(t) \leqq(2 \log t)^{1 / 2}+(1 / \alpha+1 / 2+\varepsilon) \log \log t /(2 \log t)^{1 / 2}\right. \\
\text { for all } \left.t \geqq t_{0}(\omega)\right)=1 .
\end{array}
$$

H. Cramér [2] and M. G. Sur [7] have obtained the results corresponding to (2.1) in the case of $\alpha=2$ when $x(t)$ is stationary.

In this case, assuming conditions (A.1), (A.1') and (A.2) and denoting $E(x(t) x(0)) /(v(0))^{2}$ by $\rho(t)$, it follows that

$$
\begin{gathered}
\exists \alpha 2 \geqq \alpha>0, \lim _{t \rightarrow \infty} \sup t^{-\alpha}(1-\rho(t))<\infty, \\
\lim _{t \rightarrow \infty} \rho(t) t=0 .
\end{gathered}
$$

Therefore the assumptions of Theorem 5.4 of J. Pickands III [6] are satisfied. Hence

$$
P\left(\eta(t)-(2 \log t)^{1 / 2} \rightarrow 0(t \rightarrow \infty)\right)=1 .
$$

They are interested in the function $\phi_{1}$ and $\phi_{2}$ such that it holds

$$
P\left(\exists t_{0}(\omega), \phi_{1}(t) \leqq \eta(t) \leqq \phi_{2}(t), \text { for all } t \geqq t_{0}(\omega)\right)=1 .
$$

We have only proposed some criteria concerning as $\phi_{2}(\cdot)$. Also, we remark that M. Nisio [5] proved under the weaker condition than (A.1), (A.1') and (A.2) that

$$
P\left(\lim _{t \rightarrow \infty} \frac{\eta(t)}{(2 \log t)^{1 / 2}}=1\right)=1 \text {. }
$$

And also, we can easily show that the stationary Gaussian process with $\rho(t)$ $=E(x(t) x(0))=\exp \left(-|t|^{\alpha}\right)(0<\alpha \leqq 2)$ satisfies the conditions (A.1), (A.1') and (A.2).

The proof of Theorems 1 and 2 will be carried out in the similar way to [8] and $[10]$ and given in $\$ \$ 3$ and 4 , respectively.

Next, we will deal with another class of Gaussian processes including the Brownian motion. We introduce, here, the following conditions. 
(C.1) There are such positive constants $\delta_{2}, C_{3}$ and $T_{3}$ as rendering the following valid

$$
\rho(t, t+h) \geqq 1-C_{3}(h / t)^{\alpha},
$$

for all $t$ and $h$ such that $0<(h / t)<\delta_{2}$ and $t>T_{3}$ for some $\alpha$ with $0<\alpha \leqq 2$.

(C.1') There are positive constants $\delta_{3}, C_{4}$ and $T_{4}$ such that

$$
\rho(t, t+h) \leqq 1-C_{4}(h / t)^{\alpha},
$$

for all $t$ and $h$ such that $0<(h / t)<\delta_{3}$ and $t>T_{4}$, and for all $t$ and $s$ such that $(h / t)>\delta_{3}$ and $t>T_{4}, \rho(t, t+h)<1-C_{4} \delta_{3}^{\alpha}$, for some $\alpha$ with $0<\alpha \leqq 2$.

(C.2) The limit

$$
\lim _{s \rightarrow \infty} \rho(t, t s) \log s=0,
$$

converges uniformly with respect to $t$.

THEOREM 4. Suppose that condition (C.1) is satisfied. If $\psi(t) \in M_{a}^{+}$and

$$
\int_{a}^{\infty} \frac{1}{t} \psi(t)^{2 / \alpha-1} \exp \left(-\frac{1}{2} \psi^{2}(t)\right) d t<\infty,
$$

for some $a>0$, then we have

$$
P\left(\exists t_{0}(\omega), x(t) \leqq v(t) \psi(t), \text { for all } t>t_{0}(\omega)\right)=1 .
$$

TheOREM 5. Suppose that (C.1') and (C.2) are satisfied. If $\psi(t) \in M_{a}^{+}$and

$$
\int_{a}^{\infty} \frac{1}{t} \psi(t)^{2 / \alpha-1} \exp \left(-\frac{1}{2} \psi^{2}(t)\right) d t=\infty
$$

for some $a>0$, then we have

$$
P(x(t)>v(t) \psi(t) \text { i.o. })=1 .
$$

Combination of Theorems 4 and 5 will lead to the following theorem and corollaries.

THEOREM 6. Assume that conditions (C.1), (C.1') and (C.2) are satisfied at the same time for some $\alpha$. Let $\psi(t) \in M_{a}^{+}$for some a. Then

$$
P\left(\exists t_{0}(\omega), x(t) \leqq v(t) \psi(t) \text { for all } t \geqq t_{0}(\omega)\right)=1 \text { or } 0,
$$

according as the integral

$$
\int_{a}^{\infty} \frac{1}{t} x(t)^{2 / \alpha-1} \exp \left(-\frac{1}{2} \psi^{2}(t)\right) d t
$$

converges or diverges.

COROllary 1. Under the same conditions as in Theorem 6 , for every $\varepsilon>0$, we have

$$
P\left(\exists t_{0}(\omega), x(t) \leqq v(t)\left(2 \log _{(2)} t+(2 / \alpha+1+\varepsilon) \log _{(3)} t\right)^{1 / 2} \quad \text { for all } t \geqq t_{0}(\omega)\right)=1 .
$$

Moreover, for any $\varepsilon \geqq 0$, we have

$$
P\left(x(t)>v(t)\left(2 \log _{(2)} t+(2 / \alpha+1-\varepsilon) \log _{(3)} t\right)^{1 / 2} \text { i.o. }\right)=1 .
$$


The proof of Theorems 4 and 5 will be carried out directly by the minor change of the proof of Theorems 1 and 2 , namely, by putting $t_{p, k}=2^{p}+2^{p} k /[\log p]^{1 / \alpha}$ in $E(p ; k)=\left\{y\left(t_{p, k}\right)>\phi\left(t_{p, k}\right)\right\}$ which is used in the proof of Theorems 1 and 2 . Here, we make an important remark: the conditions (C.1) (C.2) can be deduced by the time change $s=e^{t}$ from the conditions (A.1) (A.2) and conversely. Therefore, by the time change, Theorems 4,5 and 6 can be deduced from Theorems 1,2 and 3 and conversely.

Some applications of Theorem 6 will be given in the following. Let $B(t)$ be a one dimensional Brownian motion on $(-\infty, \infty)$. We can easily show that it satisfies the conditions (C.1) (C.2). Therefore, Theorem 6 contains as a special case Theorem A. Furthermore, we consider the process $X=\left\{x(t)=\int_{0}^{t} B(u) d u\right.$, $-\infty<t<\infty\}$. Then the process $X$ is a Gaussian process with $E(x(t))=0$. Suppose that $0<s<t$, then, we have $r(t, s)=E(x(t) x(s))=s^{2} t / 2-s^{3} / 6$. Furthermore, if $0<t<t+h$,

$$
\rho(t, t+h)=\frac{1+\frac{3}{2}(h / t)}{(1+h / t)^{3 / 2}} \sim 1-\frac{3}{8}\left(\frac{h}{t}\right)^{2} \quad\left(\frac{h}{t} \sim 0\right) .
$$

Hence, there is positive constant $\delta$ such that there holds

$$
1-\frac{1}{2}(h / t)^{2}<\rho(t, t+h)<1-\frac{1}{4}(h / t)^{2},
$$

for all $t$ and $h$ such that $0<h / t<\delta$. Therefore, from this and (2.2), we can show that the conditions (C.1) and (C.1') hold for the process $X$. Next,

$$
\rho(t, t s) \log s=\frac{1+\frac{3}{2}(s-1)}{\left(s^{3}\right)^{1 / 2}} \log s \rightarrow 0 \quad(s \rightarrow \infty),
$$

which justifies the condition (C.2). Accordingly, Theorem 6 is applicable to the present case.

THEOREM 7. Let $B(t)$ be the Brownian motion. Let $\psi(t) \in M_{a}^{+}$, for some a>0. Then,

$$
P\left(\exists T_{0}(\omega), \int_{0}^{T} B(u) d u \leqq\left(\frac{T^{3}}{3}\right)^{1 / 2} \psi(T) \text { for all } T>T_{0}(\omega)\right)=1 \text { or } 0
$$

according as the integral

$$
\int_{a}^{\infty} \frac{1}{t} \exp \left(-\frac{1}{2} \psi^{2}(t)\right) d t
$$

converges or diverges.

Corollary 1. For every $\varepsilon>0$,

$$
\begin{array}{r}
P\left(\exists T_{0}(\omega), \int_{0}^{T} B(u) d u \leqq\left(\frac{T^{3}}{3}\right)^{1 / 2}\left(2 \log _{(2)} T+2 \log _{(3)} T+\cdots(2+\varepsilon) \log _{(n)} T\right)^{1 / 2}\right. \\
\text { for all } \left.T>T_{0}(\omega)\right)=1 .
\end{array}
$$


While for any $\varepsilon \geqq 0$,

$$
P\left(\int_{0}^{T} B(u) d u>\left(\frac{T^{3}}{3}\right)^{1 / 2}\left(2 \log _{(2)} T+2 \log _{(3)} T+\cdots(2-\varepsilon) \log _{(n)} T\right)^{1 / 2} \text { i.o. }\right)=1 .
$$

\section{Corollary 2.}

$$
P\left(\limsup _{T \rightarrow \infty}\left[\int_{0}^{T} B(u) d u /\left(\frac{2}{3} T^{3} \log _{(2)} T\right)^{1 / 2}\right]=1\right)=1 .
$$

\section{Proof of Theorem 1.}

LEMMA 3.1. If Theorem 1 is true under the additional condition that for large $t$,

$$
(\log t)^{1 / 2} \leqq \phi(t) \leqq(3 \log t)^{1 / 2},
$$

it is true without the additional condition.

Proof. The proof of Lemma 3.1 is similar to Lemma 1 in T. Sirao [8]. Therefore, we will give only its sketch.

Assume that the statement of Theorem 1 is true for arbitrary $\phi$ such that

$$
\int_{a}^{\infty} \phi(t)^{2 / \alpha-1} \exp \left(-\frac{1}{2} \phi^{2}(t)\right) d t<\infty \text { for some } a>0
$$

and

$$
(\log t)^{1 / 2} \leqq \phi(t) \leqq(3 \log t)^{1 / 2}
$$

for large $t$.

Given any $\phi(t)$ satisfying only (3.1), the set $\left\{t ; \phi(t)<(\log t)^{1 / 2}\right\}$ is bounded. For, if it is not, there are an infinite set $\left\{t_{n}\right\}$ such that $\phi\left(t_{n}\right)<\left(\log t_{n}\right)^{1 / 2}$. Then we can easily show that

$$
\int_{t_{1}}^{\infty} \phi(t)^{2 / \alpha-1} \exp \left(-\frac{1}{2} \phi^{2}(t)\right) d t \geqq\left(\frac{\left(t_{n}-t_{1}\right)^{2}}{t_{n}}\right)^{1 / 2}\left(\log t_{n}\right)^{1 / \alpha-1 / 2} \rightarrow \infty \quad(n \rightarrow \infty),
$$

which contradicts to (3.1).

Now put $\hat{\phi}(t)=\min \left(\max \left(\phi(t),(\log t)^{1 / 2}\right),(3 \log t)^{1 / 2}\right)$. Then,

$$
\hat{\phi}(t)=\min \left(\phi(t),(3 \log t)^{1 / 2}\right)
$$

for large $t$. Hence

$$
\int^{\infty} \hat{\phi}(t)^{2 / \alpha-1} \exp \left(-\frac{1}{2} \hat{\phi}^{2}(t)\right) d t<\infty
$$

By definition, $(\log t)^{1 / 2} \leqq \hat{\phi}(t) \leqq(3 \log t)^{1 / 2}$. Thus, Theorem 1 is valid for $\hat{\phi}(t)$, that is to say,

$$
P\left(\exists t_{0}(\omega), x(t) \leqq v(t) \hat{\phi}(t), \text { for all } t \geqq t_{0}(\omega)\right)=1 .
$$

Recalling that $\hat{\phi}(t) \leqq \phi(t)$ for large $t$, we have the conclusion of Lemma 3.1. 
By Lemma 3.1, it is sufficient to show Theorem 1, under the hypothesis (3.1) and (3.2). In the following, we put $y(t)=x(t) / v(t)$.

LEMMA 3.2. Let $\phi(t)$ satisfy (3.1) and let $E(p ; k)=\left\{y\left(t_{p, k}\right)>\phi\left(t_{p, k}\right)\right\}$, where $t_{p, k}=p+k /\left[(\log p)^{1 / \alpha}\right],\left(p=1, \ldots, k=0,1,2, \ldots,\left[(\log p)^{1 / \alpha}\right]\right)$. Then,

$$
\sum_{p=1}^{\infty} \sum_{k=0}^{\left[(\log p)^{1 / \alpha}\right]} P(E(p ; k))<\infty \text {. }
$$

Proof. Since

$$
\int_{x}^{\infty} e^{-u^{2} / 2} d u \leqq \frac{1}{x} e^{-x^{2} / 2} \text { for any } x>0
$$

we have for any $p_{0}$,

$$
\sum_{p=p_{0}}^{\infty} \sum_{k=0}^{\left[(\log p)^{1 / \alpha}\right]} P(E(p ; k)) \leqq \frac{1}{(2 \pi)^{1 / 2}} \sum_{p=p_{0}}^{\infty} \sum_{k=0}^{\left[(\log p)^{1 / \alpha}\right]} \frac{1}{\phi\left(t_{p, k}\right)} \exp \left(-\frac{1}{2} \phi^{2}\left(t_{p, k}\right)\right) \equiv A .
$$

By use of the monotonicity of $\phi(\cdot)$, we have

$$
A \leqq(2 \pi)^{-1 / 2} \sum_{p=p_{0}}^{\infty}(\log p)^{1 / \alpha} \frac{1}{\phi(p)} \exp \left(-\frac{1}{2} \phi^{2}(p)\right) \equiv B .
$$

Since $(\log p)^{1 / 2} \leqq \phi(p)$ for large $p$, if we take sufficiently large $p_{0}$, we obtain

$$
\begin{aligned}
B & \leqq(2 \pi)^{-1 / 2} \sum_{p=p_{0}}^{\infty} \phi(p)^{2 / \alpha-1} \exp \left(-\frac{1}{2} \phi^{2}(p)\right) \\
& \leqq(2 \pi)^{-1 / 2} \int_{p_{0}}^{\infty} \phi(t)^{2 / \alpha-1} \exp \left(-\frac{1}{2} \phi^{2}(t)\right) d t<\infty,
\end{aligned}
$$

which concludes the proof.

Let $L$ be any positive constant. Let $c$ be a real number which makes $e^{q c}$ an integer for any positive integer $q$. Let $t_{p, k, q, m}=t_{p, k}+m /\left(e^{q c}\left[(\log p)^{1 / \alpha}\right]\right)$ and let

$$
F_{q, m}(p ; k)=y\left(t_{p, k, q, m}\right) \geqq \phi\left(t_{p, k}\right)+\frac{2 L}{\phi\left(t_{p, k}\right)} \sum_{i=0}^{q-1} 2^{-\alpha i}
$$

where the integers $m$ range from 0 to $e^{q c}$.

Let $\bigcup_{m=0}^{q a c} F_{q, m}(p ; k)=F_{q}(p ; k)$ and let $\bigcup_{q=0}^{\infty} F_{q}(p ; k)=F(p ; k)$. Then, we have, for almost all $y(\cdot, \omega)$,

$$
F(p ; k)=\left\{y\left(t_{p, k}+s /\left[(\log p)^{1 / \alpha}\right]\right) \geqq \phi\left(t_{p, k}\right)+\frac{2 L}{\phi\left(t_{p, k}\right)} \sum_{i=0}^{\infty} 2^{-\alpha i} \text { for some } 0 \leqq s \leqq 1\right\},
$$

since almost all path functions $y(t, \omega)$ are continuous.

LEMMA 3.3.

$$
\sum_{p=1}^{\infty} \sum_{k=0}^{\left[(\log p)^{1 / \alpha}\right]} P(F(p ; k))<+\infty
$$


Before proving this lemma, we will use it to carry out the proof of Theorem 1. By use of the Borel-Cantelli theorem, it follows that

$$
\begin{aligned}
& P\left(\exists p_{0}, \forall p \geqq p_{0}, 0 \leqq \forall s \leqq 1,1 \leqq \forall k \leqq\left[(\log p)^{1 / \alpha}\right], y\left(t_{p, k}+s /\left[(\log p)^{1 / \alpha}\right]\right)\right. \\
&\left.\leqq \phi\left(t_{p, k}\right)+\frac{2 L}{\phi\left(t_{p, k}\right)} \sum_{i=0}^{\infty} 2^{-\alpha i}\right)=1 .
\end{aligned}
$$

By the monotonicity of $\phi$, we find

$$
P\left(\exists T_{0}(\omega), y(T) \leqq \phi(T)+\frac{2 L}{\phi(T)} \sum_{i=0}^{\infty} 2^{-\alpha i}, \text { for all } T \geqq T_{0}(\omega)\right)=1 .
$$

Let $\phi(t)=\phi(t)-3 L^{\prime} / \phi$, where $L^{\prime}=L \sum_{i=0}^{\infty} 2^{-\alpha i}$. Then, for sufficiently large $t$, it holds that

$$
(a \log t)^{1 / 2} \leqq \phi(t) \leqq(3 \log t)^{1 / 2}
$$

with some $0<a<1$, and

$$
\int^{\infty} \phi(t)^{2 / \alpha-1} \exp \left(-\frac{1}{2} \phi^{2}(t)\right) d t<\infty
$$

Since we can repeat the similar discussion for this $\phi$, we can obtain that

$$
P\left(\exists T_{0}(\omega), y(T) \leqq \phi(T)+2 L^{\prime} / \phi(T) \text { for all } T>T_{0}(\omega)\right)=1
$$

Since $\phi(T)+2 L^{\prime} / \phi(T)<\phi(T)$ for sufficiently large $T$, we have the conclusion of Theorem 1.

Proof of Lemma 3.3. In the following, we will sometimes denote simply $F_{q, m}$ $F_{q}$ and $F$ in place of $F_{q, m}(p ; k), F_{q}(p ; k)$ and $F(p ; k)$ respectively. Since the relation $F_{q-1} \cap F_{q}=F_{q-1}$, we have

$$
\begin{aligned}
P\left(F_{q}\right) & =P\left(F_{q-1}\right)+P\left(F_{q-1}^{\prime} \cap F_{q}\right) \\
& \leqq P\left(F_{q-1}\right)+\sum_{m=0}^{e q c} P\left(F_{q-1}^{\prime} \cap F_{q, m}\right) \\
& \leqq P\left(F_{q-1}\right)+\sum_{m=0}^{e q c} P\left(F_{q-1, m_{1}}^{\prime} \cap F_{q, m}\right)
\end{aligned}
$$

for any integer $m_{1}$ with $0 \leqq m_{1} \leqq e^{(q-1) c}$, where $F^{\prime}$ means the complementary set of $F$.

LEMMA 3.4. If we take $m_{1}$ satisfying the relation $\left|m e^{-q c}-m_{1} e^{-(q-1) c}\right|<e^{-(q-1) c}$ there exists a positive constant $C_{8}$ independent of $p, k$ and $q$ such that

$$
P\left(F_{q-1, m_{1}}^{\prime} \cap F_{q, m}\right) \leqq C_{8} e^{-2 q c} P(E(p ; k)) .
$$


Proof of Lemma 2.4. From the definition of set $F_{q, m}$, we can write

$$
\begin{aligned}
Q & \equiv P\left(F_{q-1, m_{1}}^{\prime} \cap F_{q, m}\right) \\
& =P\left(y\left(t_{p, k, q-1, m}\right) \leqq \phi\left(t_{p, k}\right)+\frac{2 L}{\phi\left(t_{p, k}\right)} \sum_{i=0}^{q-2} 2^{-\alpha i},\right. \\
& \left.y\left(t_{p, k, q, m}\right)>\phi\left(t_{p, k}\right)-\frac{2 L}{\phi\left(t_{p, k}\right)} \sum_{i=0}^{q-1} 2^{-\alpha i}\right) .
\end{aligned}
$$

Now, let $X$ and $Y$ be two independent random variables obeying the Gaussian distribution with the mean zero and variance 1 , and let $\rho_{m_{1}, m}$ be the correlation coefficient between $y\left(t_{p, k, q-1, m_{1}}\right)$ and $y\left(t_{p, k, q, m}\right)$. Then

$$
\begin{aligned}
Q \equiv & P\left(\left(1-\rho_{m_{1}, m}^{2}\right)^{1 / 2} Y+\rho_{m_{1}, m} X \leqq\left(\phi+(2 L / \phi) \sum_{i=0}^{q-2} 2^{-\alpha i}\right), X \geqq\left(\phi+(2 L / \phi) \sum_{i=0}^{q-1} 2^{-\alpha i}\right)\right) \\
\leqq & P\left(X \geqq\left(\phi+(2 L / \phi) \sum_{i=0}^{q-1} 2^{-\alpha i}\right),\left(1-\rho_{m_{1}, m}^{2}\right)^{1 / 2} Y\right. \\
\leqq & \left.\left(\phi+(2 L / \phi) \sum_{i=0}^{q-2} 2^{-\alpha i}\right)-\rho_{m_{1}, m}\left(\phi+(2 L / \phi) \sum_{i=0}^{q-1} 2^{-\alpha i}\right)\right) \\
= & P\left(X \geqq\left(\phi+(2 L / \phi) \sum_{i=0}^{q-1} 2^{-\alpha i}\right)\right) \\
& \times P\left(Y>-\left(1-\rho_{m_{1}, m}^{2}\right)^{-1 / 2}\left(1-\rho_{m_{1}, m}\right)\left(\phi+(2 L / \phi) \sum_{i=0}^{q-2} 2^{-\alpha i}\right)\right. \\
& \left.+\left(1-\rho_{m_{1}, m}^{2}\right)^{-1 / 2} \rho_{m_{1}, m}(2 L / \phi) 2^{-\alpha(q-1)}\right),
\end{aligned}
$$

where $\phi=\phi\left(t_{p, k}\right)$. By use of assumption (A.1), for large $p$,

$$
\begin{aligned}
\rho_{m_{1}, m} & \geqq 1-C_{1}\left(\frac{\left|m_{1} / e^{(q-1) c}-m / e^{q c}\right|}{\left[(\log p)^{1 / \alpha}\right]}\right)^{\alpha} \\
& \geqq 1-C_{5} /\left((\log p) e^{(q-1) \alpha}\right),
\end{aligned}
$$

where $C_{5}$ is an absolute positive constant. Taking account of this estimate and (3.2),

$$
-\left(1-\rho_{m_{1}, m}^{2}\right)^{-1 / 2}\left(1-\rho_{m_{1}, m}\right)\left(\phi+(2 L / \phi) \sum_{i=0}^{q-1} 2^{-\alpha i}\right) \geqq-C_{6},
$$

where $C_{6}$ is a positive constant independent of $p$ and $q$. Also, for a convenient positive constant $C_{7}$,

$$
\frac{\rho_{m_{1}, m}}{\left(1-\rho_{m_{1}, m}^{2}\right)^{1 / 2}} \frac{2 L}{\phi} \frac{1}{2^{\alpha(q-1)}}>C_{7}\left(\frac{e^{c}}{4}\right)^{\alpha(q-1)} .
$$

Now, we select $c$ such that $C_{7}\left(e^{c} / 4\right)^{\alpha(q-1)}>2 C_{6}$ for all $q>2$ and $\left(e^{c} / 4\right)>1$. Then we have

$$
\begin{aligned}
Q & \leqq P(X \geqq \phi) P\left(Y \geqq\left(C_{7} / 2\right)\left(e^{c} / 4\right)^{(q-1) \alpha / 2}\right) \\
& \leqq P(E(p ; k))(2 \pi)^{-1 / 2} \frac{2}{C_{7}\left(e^{c} / 4\right)^{(q-1) \alpha / 2}} \exp \left(-\frac{1}{2}\left(\frac{C_{7}^{2}}{4}\right)\left(\frac{e^{c}}{4}\right)^{(q-1) \alpha}\right) .
\end{aligned}
$$


Since

$$
\lim _{q \rightarrow \infty} e^{2 q c} \frac{1}{\left(e^{c} / 4\right)^{(q-1) \alpha / 2}} \exp \left(-\frac{1}{2}\left(\frac{C_{7}^{2}}{4}\right)\left(\frac{e^{c}}{4}\right)^{(q-1) \alpha}\right)=0,
$$

the above expression is smaller than the following quantity, $C_{8} e^{-2 q c} P(E(p ; k))$, where $C_{8}$ is an absolute constant independent of $p$ and $q$. This concludes the proof.

From Lemma 3.4, it follows that

$$
\begin{aligned}
P\left(F_{q}\right) & \leqq P\left(F_{q-1}\right)+C_{8} e^{-q c} P(E(p ; k)) \\
& \leqq C_{8} \sum_{i=0}^{q-1} e^{-i c} P(E(p ; k)) .
\end{aligned}
$$

When $q \rightarrow \infty$,

$$
P(F(p ; k)) \leqq C_{8} \sum_{i=0}^{\infty} e^{-i c} P(E(p ; k))=\text { Constant } \times P(E(p ; k)) .
$$

Hence, for sufficiently large $\tilde{p}$,

$$
\sum_{p=\tilde{p}}^{\infty} \sum_{k=0}^{\left[(\log p)^{1 / \alpha}\right]} P(F(p ; k)) \leqq \text { Constant } \times \sum_{p=0}^{\infty} \sum_{k=0}^{\left[(\log p)^{1 / \alpha}\right]} P(E(p ; k)),
$$

which is convergent by Lemma 3.2. This concludes the proof of Lemma 3.3.

\section{Proof of Theorem 2.}

LEMмA 4.1. If Theorem 2 is true under the additional assumption that for large $t$,

$$
(\log t)^{1 / 2} \leqq \phi(t) \leqq(3 \log t)^{1 / 2},
$$

it is true without the additional assumption.

Proof. The proof of Lemma 4.1 is similar to Lemma 1 in T. Sirao [8]. We assume that Theorem 2 is true under the additional assumption. Let $\phi(t)$ be an arbitrary function with $\int^{\infty} \phi(t)^{2 / \alpha-1} \exp \left(-\frac{1}{2} \phi^{2}(t)\right) d t=\infty$. Let

$$
\hat{\phi}(t)=\min \left(\max (\phi(t),(\log t))^{1 / 2},(3 \log t)^{1 / 2}\right) .
$$

Suppose that there exists an infinite sequence $\left\{t_{n}\right\}$ such that $\phi\left(t_{n}\right)<\left(\log t_{n}\right)^{1 / 2}$ and $t_{n} \rightarrow \infty(n \rightarrow \infty)$. Then, $\int^{\infty} \hat{\phi}(t)^{2 / \alpha-1} \exp \left(-\frac{1}{2} \hat{\phi}^{2}(t)\right) d t=\infty$, because in this case $\hat{\phi}\left(t_{n}\right)=\left(\log t_{n}\right)^{1 / 2}$ and therefore,

$$
\begin{aligned}
\int_{t_{1}}^{\infty} \hat{\phi}(t)^{2 / \alpha-1} \exp \left(-\frac{1}{2} \hat{\phi}^{2}(t)\right) d t & \geqq \int_{t_{1}}^{t_{n}} \hat{\phi}(t)^{2 / \alpha-1} \exp \left(-\frac{1}{2} \hat{\phi}^{2}(t)\right) d t \\
& \geqq\left(t_{n}-t_{1}\right) \hat{\phi}\left(t_{n}\right)^{2 / \alpha-1} \exp \left(-\frac{1}{2} \hat{\phi}^{2}\left(t_{n}\right)\right) \\
& =\frac{t_{n}-t_{1}}{\left(t_{n}\right)^{1 / 2}}\left(\log t_{n}\right)^{1 / \alpha-1 / 2} .
\end{aligned}
$$

Next, suppose that $\phi(t)>(\log t)^{1 / 2}$ for large $t$. In this case,

$$
\min \left(\phi(t),(3 \log t)^{1 / 2}\right)=\hat{\phi}(t) .
$$


Therefore, for all large $t$,

Hence,

$$
\phi(t)^{2 / \alpha-1} \exp \left(-\frac{1}{2} \phi^{2}(t)\right) \leqq \hat{\phi}(t)^{2 / \alpha-1} \exp \left(-\frac{1}{2} \hat{\phi}^{2}(t)\right)
$$

$$
\int^{\infty} \hat{\phi}(t)^{2 / \alpha-1} \exp \left(-\frac{1}{2} \hat{\phi}^{2}(t)\right) d t=\infty
$$

Furthermore,

$$
(\log t)^{1 / 2} \leqq \hat{\phi}(t) \leqq(3 \log t)^{1 / 2} .
$$

Consequently, by assumption of Lemma 4.1,

$$
P\left(\exists\left\{T_{n}(\omega)\right\}, T_{1}<T_{2}<\cdots<T_{n} \rightarrow \infty(n \rightarrow \infty), y\left(T_{n}\right)>\hat{\phi}\left(T_{n}\right)\right)=1 .
$$

But, by Theorem 1, clearly,

Therefore,

$$
P\left(\left(3 \log T_{n}(\omega)^{1 / 2}\right)>y\left(T_{n}(\omega)\right) \forall n>\exists n_{0}(\omega)\right)=1 .
$$

Hence,

$$
P\left(\left(3 \log T_{n}\right)^{1 / 2}>\hat{\phi}\left(T_{n}\right), \forall n>\exists n_{0}(\omega)\right)=1
$$

Thus

$$
P\left(\hat{\phi}\left(T_{n}\right)=\max \left(\phi\left(T_{n}\right),\left(\log T_{n}\right)^{1 / 2}\right)>\phi\left(T_{n}\right), \forall n>\exists n_{0}(\omega)\right)=1 .
$$

$$
P\left(y\left(T_{n}\right)>\phi\left(T_{n}\right), \forall n>\exists n_{0}(\omega)\right)=1,
$$

which concludes the proof.

As in $\S 2$, we consider the family of events $E(p ; k)$, where $p$ ranges over the positive integers and integer $k$ ranges over $0 \leqq k \leqq\left[(\log p)^{1 / \alpha}\right]$. We enumerate events $E(p ; k)$ such that $n<n^{\prime}$ if and only if $p+k /\left[(\log p)^{1 / \alpha}\right]<p^{\prime}+k^{\prime} /\left[\left(\log p^{\prime}\right)^{1 / \alpha}\right]$ if we put $E_{n}=E(p ; k)$ and $E_{n^{\prime}}=E\left(p^{\prime} ; k^{\prime}\right)$. Then, the proof of Theorem 2 is carried out by proving the following sequence of lemmas. For, then, by Chung-Erdös [1] we can see that $P\left(E_{n}\right.$ i.o. $)=1$.

LEMMA 4.2. $\sum_{n=1}^{\infty} P\left(E_{n}\right)=+\infty$.

LeMmA 4.3. For any pair of $(n, h)$ with $n \geqq h$, there exist $c(h)>0$ and $H(n, h)>n$ such that for any $m \geqq H(n, h)$

$$
P\left(E_{m} \mid E_{h}^{\prime} \cap E_{h+1}^{\prime} \cap E_{h+2}^{\prime} \cap \cdots \cap E_{n}^{\prime}\right) \geqq c(h) P\left(E_{m}\right) .
$$

Lemma 4.4. There exist two absolute constants $K_{1}$ and $K_{2}$ with the following property: to each $E_{n}$ there corresponds a set of events $E_{n_{1}}, E_{n_{2}}, \ldots, E_{n_{s}}$ belonging to $\left\{E_{n}\right\}$ such that

$$
\sum_{i=1}^{s} P\left(E_{n} \cap E_{n_{i}}\right)<K_{1} P\left(E_{n}\right)
$$

and that for any other $E_{m}$ than $E_{n_{i}}(1 \leqq i \leqq s)$ which stands after $E_{n}$ in the sequence (viz. $m>n$ ) the inequality

$$
P\left(E_{n} \cap E_{m}\right) \leqq K_{2} P\left(E_{n}\right) P\left(E_{m}\right)
$$

holds. 
Proof of Lemma 4.2. Since $P\left(E_{n}\right)=P(E(p ; k))$, it is sufficient to show that

$$
\sum_{p=p_{0}}^{\infty} \sum_{k=0}^{\left[(\log p)^{1 / \alpha}\right]} P(E(p ; k))=\infty
$$

for some $p_{0}$. The left hand side of the above equality is greater than

$$
\sum_{p=p_{0}}^{\infty} \sum_{k=0}^{\left[(\log p)^{1 / \alpha}\right]} 2^{-1}(2 \pi)^{-1 / 2} \frac{1}{\phi\left(t_{p, k}\right)} \exp \left(-\frac{1}{2} \phi^{2}\left(t_{p, k}\right)\right) \equiv R,
$$

because $\int_{x}^{\infty} e^{-u^{2} / 2} d u \geqq(1 / 2 x) e^{-x^{2} / 2}(x \geqq 1)$. By the monotonicity of the function $1 / \phi(x) \exp \left(-\frac{1}{2} \phi^{2}(x)\right)$ of $x$ for large $x$,

$$
\begin{aligned}
R & \geqq 2^{-1}(2 \pi)^{-1 / 2} \sum_{p=p_{0}}^{\infty}[(\log p)]^{1 / \alpha} \frac{1}{\phi(p+1)} \exp \left(-\frac{1}{2} \phi^{2}(p+1)\right) \\
& \geqq\left(\frac{\log p_{0}}{\log \left(p_{0}+1\right)}\right)^{1 / \alpha} \frac{1}{3^{1 / \alpha}} 2^{-1}(2 \pi)^{-1 / 2} \sum_{p=p_{0}}^{\infty} \phi(p+1)^{2 / \alpha-1} \exp \left(-\frac{1}{2} \phi^{2}(p+1)\right) \\
& \geqq \text { Constant } \times \int_{p_{0}+1}^{\infty} \phi(t)^{2 / \alpha-1} \exp \left(-\frac{1}{2} \phi^{2}(t)\right) d t,
\end{aligned}
$$

which concludes the proof.

Proof of Lemma 4.3. The proof is similar to the discussion in pp. 146-148 of T. Sirao [8]. Therefore, we will give only an outline of it. Let

$$
F_{n}(a)=\left\{\omega ; \phi\left(t_{p, k}\right) \leqq y\left(t_{p, k}\right) \leqq \phi\left(t_{p, k}\right)+a\right\} .
$$

Then, for large $n$,

$$
P\left(E_{n}\right) \geqq P\left(F_{n}(a)\right) \geqq \frac{1}{2} P\left(E_{n}\right)\left(1-2 \exp \left[-a \phi\left(t_{p, k}\right)\right]\right) .
$$

Therefore, given a pair of $(n, h)$, we can take $a>0$ such that

$$
P\left(F_{m}(a)\right) \geqq \frac{1}{3} P\left(E_{m}\right) \quad \text { for all } m \geqq n
$$

and

$$
P\left(\bigcap_{i=h}^{n}\left(E_{i}^{\prime} \cap E_{i}(a)\right)\right) \geqq \frac{1}{2} P\left(\bigcap_{i=h}^{n} E_{i}^{\prime}\right),
$$

where $E_{n}(a)=\left\{\omega ; y\left(t_{p, k}\right)+a \geqq 0\right\}$. Hence, we have

$$
P\left(E_{m} \mid E_{h}^{\prime} \cap E_{h+1}^{\prime} \cap \cdots \cap E_{n}^{\prime}\right) \geqq \frac{1}{2} P\left(F_{m}(a) \mid \bigcap_{i=h}^{n}\left(E_{i}^{\prime} \cap E_{i}(a)\right)\right) .
$$

Let

$$
\rho_{i, m}=\rho\left(y\left(p_{i}+k_{i} /\left[\left(\log p_{i}\right)^{1 / \alpha}\right]\right), \quad y\left(p_{m}+k_{m} /\left[\left(\log p_{m}\right)^{1 / \alpha}\right]\right)\right)
$$

and $\rho_{m}=\max \left\{\left|\rho_{i, m}\right| ; 0 \leqq i \leqq n-m\right\}$. Then by condition (A.2), we can see that for large $m$,

$$
\rho_{m}^{-1 / 2} \geqq 4\left(\log p_{m}\right)^{1 / 2} \geqq \phi\left(p_{m}+k_{m} /\left[\left(\log p_{m}\right)^{1 / \alpha}\right]\right)
$$

and $\lim _{m \rightarrow \infty} \rho_{m}=0$. 
Now, let $X_{i}=y\left(p_{i}+k_{i} /\left[\left(\log p_{i}\right)^{1 / \alpha}\right]\right)$. Let $A_{i}=\left[-a, \phi\left(p_{i}+k_{i} /\left[\log p_{i}\right]^{1 / \alpha}\right)\right)$ for $i=0$, $1,2, \ldots, n-h$. Let $Y_{m}=y\left(p_{m}+k_{m} /\left[\left(\log p_{m}\right)^{1 / \alpha}\right]\right)$ and $B_{m}=\left[\phi\left(p_{m}+k_{m} /\left[\left(\log p_{m}\right)^{1 / \alpha}\right]\right)\right.$, $\left.\phi\left(p_{m}+k_{m} /\left[\left(\log p_{m}\right)^{1 / \alpha}\right]\right)+a\right)$, where $m=n+1, n+2, \ldots$ Then, the following lemma is available (Lemma 4 in T. Sirao [8]).

Lemma 4.5. Let $\left\{X_{1}, X_{2}, \ldots, X_{n}, Y_{m}, m=1,2, \ldots\right\}$ be a sequence of standard Gaussian random variables and $\rho_{i, m}=E\left(X_{i} Y_{m}\right), 0 \leqq i \leqq k$ and $m \geqq 1$. We assume that $\rho_{m}=\max \left\{\left|\rho_{i, m}\right| ; 0 \leqq i \leqq k\right\} \rightarrow 0$ as $m \rightarrow \infty$ and $A_{i}(i=1,2, \ldots, k)$ are bounded Borel sets. Then, for any sequence of Borel sets $B_{m} \subset\left[-\rho_{m}^{-c}, \rho_{m}^{-c}\right]$ with $c<1$, we have

$$
\frac{P\left(Y_{m} \in B_{m} \mid X_{i} \in A_{i}, i=0,1,2, \ldots, k\right)}{P\left(Y_{m} \in B_{m}\right)} \rightarrow 1 \quad(m \rightarrow \infty) .
$$

Proof. We will give only its sketch. Denoting by $P_{X}$ the probability law of $\left\{X_{1}, X_{2}, \ldots, X_{n}\right\}$ and by $p_{m}\left(X_{1}, X_{2}, \ldots, X_{n}\right)$ the conditional expectation of $Y_{m}$ for given values of $X_{1}, X_{2}, \ldots, X_{n}$, respectively, we have

$$
\begin{aligned}
& P\left(X_{i} \in A_{i}, i=1,2, \ldots, Y_{m} \in B_{m}\right) \\
& =\int_{x_{1} \in A_{1}} \cdots \int_{x_{n} \in A_{n}}\left\{\int_{z \in B_{m}} \frac{1}{\left(2 \pi\left(1-\gamma^{2}\right)^{1 / 2}\right)} \exp \left(-\frac{1}{2\left(1-\gamma^{2}\right)}\left(z-p\left(x_{1}, x_{2}, \ldots, x_{n}\right)\right)^{2}\right) d z\right\} \\
& \cdot P_{X}\left(d x_{1}, \ldots, d x_{n}\right) \\
& =\int_{x_{1} \in A_{1}} \cdots \int_{x_{n} \in A_{n}}\left\{\int_{z \in B_{m}} \frac{1}{\left(2 \pi\left(1-\gamma^{2}\right)^{1 / 2}\right)} \exp \left(-\frac{1}{2} z^{2}+\theta\right) d z\right\} P_{X}\left(d x_{1}, \ldots, d x_{n}\right),
\end{aligned}
$$

where

$$
\theta=-\left\{\gamma^{2} z^{2}-2 z p_{m}\left(x_{1}, \ldots, x_{n}\right)+p_{m}^{2}\left(x_{1}, \ldots, x_{n}\right)\right\} /\left(2\left(1-\gamma^{2}\right)\right)
$$

and $\gamma^{2}=E\left(p_{m}^{2}\left(X_{1}, \ldots, X_{n}\right)\right)$. From the assumptions of the lemma, we can easily infer that $\gamma^{2}$ and $p_{m}$ tend to zero uniformly with respect to $x_{1}, \ldots, x_{n}$ and $z$ as $m$ tends to infinity, giving the conclusion of the lemma.

Thus we have

$$
P\left(F_{m}(a) \mid \bigcap_{i=h}^{n}\left(E_{i}^{\prime} \cap E_{i}(a)\right) \geqq \frac{1}{2} P\left(F_{n}(a)\right)\right) .
$$

By combining (4.1), (4.2), and (4.3), we obtain

$$
P\left(E_{m} \mid E_{h}^{\prime} \cap E_{h+1}^{\prime} \cap \cdots \cap E_{n}^{\prime}\right) \geqq P\left(E_{m}\right) / 12 \text { for large } m,
$$

which concludes the proof.

Proof of Lemma 4.4. In the following, if $E_{n}=E(p ; k)$ and $E_{n^{\prime}}=E\left(p^{\prime} ; k^{\prime}\right)$, we put $t_{p, k}=t_{n}$ and $t_{p^{\prime}, k^{\prime}}=t_{n^{\prime}}$. Let $A_{n}=\left\{n^{\prime} ; \rho\left(t_{n}, t_{n^{\prime}}\right)<\left(\phi\left(t_{n}\right) \phi\left(t_{n^{\prime}}\right)\right)^{-1}\right\}$ for each fixed $n$. Let us take an arbitrary $n^{\prime}$ in $A_{n}$. Then

$$
\begin{aligned}
P\left(E_{n} \cap E_{n^{\prime}}\right) & =P\left(y\left(t_{n}\right) \geqq \phi\left(t_{n}\right), y\left(t_{n^{\prime}}\right) \geqq \phi\left(t_{n^{\prime}}\right)\right) \\
& \leqq \text { Constant } \times P\left(E_{n}\right) P\left(E_{n^{\prime}}\right) .
\end{aligned}
$$

In fact, if we denote, by $X$ and $Y$ two independent standard Gaussian random variables, we can write

$$
P\left(E_{n} \cap E_{n^{\prime}}\right)=P\left(X \geqq \phi\left(t_{n^{\prime}}\right), \rho X+\left(1-\rho^{2}\right)^{1 / 2} Y \geqq \phi\left(t_{n}\right)\right),
$$


where $\rho=\rho\left(t_{n}, t_{n^{\prime}}\right)$. In case of $\rho \leqq 0$, we have

$$
\begin{aligned}
P\left(E_{n} \cap E_{n^{\prime}}\right) & \leqq P\left(X \geqq \phi\left(t_{n^{\prime}}\right), Y \geqq \phi\left(t_{n}\right) /\left(1-\rho^{2}\right)^{1 / 2}\right) \\
& \leqq P\left(X \geqq \phi\left(t_{n^{\prime}}\right)\right) P\left(Y \geqq \phi\left(t_{n}\right)\right) \\
& =P\left(E_{n^{\prime}}\right) P\left(E_{n}\right) .
\end{aligned}
$$

Next, we consider the case of $0<\rho \leqq 1 /\left(\phi\left(t_{n}\right) \phi\left(t_{n^{\prime}}\right)\right)$. At first, we divide it into three parts as follows

$$
\begin{aligned}
P\left(E_{n} \cap E_{n^{\prime}}\right)= & P\left(\phi\left(t_{n^{\prime}}\right) \leqq y\left(t_{n^{\prime}}\right) \leqq 2 \phi\left(t_{n^{\prime}}\right), 2 \phi\left(t_{n^{\prime}}\right) \geqq y\left(t_{n}\right) \geqq \phi\left(t_{n}\right)\right) \\
& +P\left(\phi\left(t_{n^{\prime}}\right) \leqq y\left(t_{n}\right), 2 \phi\left(t_{n^{\prime}}\right) \leqq y\left(t_{n^{\prime}}\right)\right) \\
& +P\left(2 \phi\left(t_{n^{\prime}}\right) \leqq y\left(t_{n^{\prime}}\right), \phi\left(t_{n^{\prime}}\right) \leqq y\left(t_{n}\right) \leqq 2 \phi\left(t_{n^{\prime}}\right)\right) .
\end{aligned}
$$

On the first term of the right hand side, we have

$$
\begin{aligned}
P\left(\phi\left(t_{n^{\prime}}\right)\right. & \left.\leqq y\left(t_{n^{\prime}}\right) \leqq 2 \phi\left(t_{n^{\prime}}\right), \phi\left(t_{n}\right) \leqq y\left(t_{n}\right) \leqq 2 \phi\left(t_{n^{\prime}}\right)\right) \\
& =P\left(\phi\left(t_{n^{\prime}}\right) \leqq X \leqq 2 \phi\left(t_{n^{\prime}}\right), \phi\left(t_{n}\right) \leqq \rho X+\left(1-\rho^{2}\right)^{1 / 2} Y \leqq 2 \phi\left(t_{n^{\prime}}\right)\right) \\
& \leqq P\left(\phi\left(t_{n^{\prime}}\right) \leqq X \leqq 2 \phi\left(t_{n^{\prime}}\right),\left(\phi\left(t_{n}\right)-2 \rho \phi\left(t_{n^{\prime}}\right)\right) /\left(1-\rho^{2}\right)^{1 / 2} \leqq Y\right) \\
& \leqq P\left(\phi\left(t_{n^{\prime}}\right) \leqq X\right) P\left(\left(\phi\left(t_{n}\right)-2 / \phi\left(t_{n^{\prime}}\right)\right) \leqq Y\right) \\
& \leqq \text { Constant } \times P\left(\phi\left(t_{n^{\prime}}\right) \leqq X\right) P\left(\phi\left(t_{n}\right) \leqq Y\right),
\end{aligned}
$$

since

$$
\lim _{n \rightarrow \infty} P\left(\phi\left(t_{n}\right)-2 / \phi\left(t_{n^{\prime}}\right) \leqq Y\right) / P\left(\phi\left(t_{n}\right) \leqq Y\right)=e^{2} .
$$

On the second term (also on the third term), we can see that

$$
\begin{aligned}
P\left(y\left(t_{n}\right)\right. & \left.\geqq \phi\left(t_{n}\right), y\left(t_{n^{\prime}}\right) \geqq 2 \phi\left(t_{n^{\prime}}\right)\right) \\
& \leqq P\left(y\left(t_{n^{\prime}}\right)>2 \phi\left(t_{n^{\prime}}\right)\right) \leqq \text { Constant } \times 1 / \phi\left(t_{n^{\prime}}\right) \exp \left(-2 \phi^{2}\left(t_{n^{\prime}}\right)\right) \\
& \leqq \text { Constant } \times P\left(E_{n}\right) P\left(E_{n^{\prime}}\right) .
\end{aligned}
$$

Next, we consider the case of $n^{\prime} \notin A_{n}$. Then, we have, for sufficiently large $n$,

$$
t_{n^{\prime}}<t_{n}+K \log t_{n}
$$

where $K$ is an absolute constant. For, from the definition, for large $n$,

$$
\rho\left(t_{n}, t_{n^{\prime}}\right) \geqq \frac{1}{\phi\left(t_{n}\right) \phi\left(t_{n^{\prime}}\right)} \geqq \frac{C_{9}}{\left(\log t_{n} \log t_{n^{\prime}}\right)^{1 / 2}},
$$

where $C_{9}$ is an absolute positive constant. Taking account of the condition (A.2), we can write

$$
\rho\left(t_{n}, t_{n^{\prime}}\right) \leqq \varepsilon\left(t_{n}, t_{n^{\prime}}\right) /\left|t_{n^{\prime}}-t_{n}\right|,
$$

where $\varepsilon\left(t_{n}, t_{n^{\prime}}\right) \rightarrow 0\left(\left|t_{n}-t_{n^{\prime}}\right| \rightarrow 0\right)$. Otherwise, suppose that there is an infinite sequence $\left\{n_{j}\right\}$ such that $t_{n_{i}}>t_{n_{j}}+K \log t_{n_{j}}$, we have, by (4.5) and (4.6),

$$
C_{9} \frac{K \log t_{n_{j}}}{\log t_{n_{j}} \log \left(t_{n_{j}}+K \log t_{n_{j}}\right)} \leqq C_{9} \frac{\left(t_{n_{j}^{\prime}}-t_{n_{j}}\right)}{\left(\log t_{n_{j}} \log t_{n_{j}^{\prime}}\right)^{1 / 2}} \leqq \varepsilon\left(t_{n_{j}}, t_{n_{j}^{\prime}}\right),
$$


from which as $j$ tends to infinity we have the contradiction $0<C_{9} K \leqq 0$, which concludes (4.4).

Therefore, we can write such as $A_{n}^{\prime}=\left\{E_{n_{i}} ; i=1,2, \ldots, s\right\}$. If $n_{i} \in A_{n}^{\prime}$,

$$
\begin{aligned}
P\left(E_{n} \cap E_{n_{\mathfrak{l}}}\right) & =P\left(y\left(t_{n}\right) \geqq \phi\left(t_{n}\right), y\left(t_{n_{\mathfrak{t}}}\right) \geqq \phi\left(t_{n_{\mathfrak{b}}}\right)\right) \\
& \leqq P\left(y\left(t_{n}\right) \geqq \phi\left(t_{n}\right), y\left(t_{n_{\mathfrak{b}}}\right) \geqq \phi\left(t_{n}\right)\right) \quad \text { for large } n,
\end{aligned}
$$

because $\phi\left(t_{n}\right) \leqq \phi\left(t_{n_{\mathfrak{t}}}\right)$. The last quantity is expressed by the following integral

$$
\frac{1}{2 \pi\left(1-\rho_{i}^{2}\right)^{1 / 2}} \int_{\phi}^{\infty} \int_{\phi}^{\infty} \exp \left(-\frac{\left(x^{2}-2 \rho_{i} x y+y^{2}\right)}{2\left(1-\rho_{i}^{2}\right)}\right) d y d x
$$

where $\phi=\phi\left(t_{n}\right)$ and $\rho_{i}=\rho\left(t_{n}, t_{n_{i}}\right)$. Rotating the axes by $\pi / 4$, we obtain

$$
\begin{aligned}
P\left(E_{n} \cap E_{n_{i}}\right) & \leqq \frac{1}{2 \pi\left(1-\rho^{2}\right)^{1 / 2}} \int_{2^{1 / 2} \phi}^{\infty} \int_{-\left(x-2^{1 / 2} \phi\right)}^{\left(x+2^{1 / 2} \phi\right)} \exp \left(-\frac{\left(1-\rho_{i}\right) x^{2}+\left(1+\rho_{i}\right) y^{2}}{2\left(1-\rho_{i}^{2}\right)}\right) d y d x \\
& \leqq(2 \pi)^{-1 / 2} \int_{\left(2 /\left(1+\rho_{i}\right)\right)^{1 / 2} \phi}^{\infty} \exp \left(-\frac{x^{2}}{2}\right) d x \\
& \leqq(2 \pi)^{-1 / 2} \phi^{-1}\left(\frac{1+\rho_{i}}{2}\right)^{1 / 2} \exp \left(-\frac{1-\rho_{i}^{2}}{2\left(1+\rho_{i}\right)^{2}} \phi^{2}\right) \exp \left(-\frac{\phi^{2}}{2}\right) \\
& \leqq 2 P\left(y\left(t_{n}\right) \geqq \phi\left(t_{n}\right)\right)\left(\frac{1+\rho_{i}}{2}\right)^{1 / 2} \exp \left(-\frac{1-\rho_{i}^{2}}{2\left(1+\rho_{i}\right)^{2}} \phi^{2}\right) \\
& \leqq 2 P\left(E_{n}\right) \exp \left(-\frac{1}{8}\left(1-\rho_{i}^{2}\right) \phi^{2}\left(t_{n}\right)\right) .
\end{aligned}
$$

Therefore,

$$
\sum_{i=1}^{s} P\left(E_{n} \cap E_{n_{i}}\right) \leqq \text { Constant } \times \sum_{i=1}^{s} \exp \left(-d\left(1-\rho_{i}^{2}\right) \phi^{2}\left(t_{n}\right)\right),
$$

where $d$ is an absolute positive constant.

In order to prove Lemma 4.4 , it is sufficient to show that $\sum_{i=1}^{s} \exp \left(-d\left(1-\rho_{i}^{2}\right) \phi^{2}\right)$ is bounded with respect to $p$. For this purpose, we shall divide it into two parts and write

$\sum_{i=1}^{s} \exp \left(-d\left(1-\rho_{i}^{2}\right) \phi^{2}\left(t_{n}\right)\right)=\sum^{(1)} \exp \left(-d\left(1-\rho_{i}^{2}\right) \phi^{2}\left(t_{n}\right)\right)+\sum^{(2)} \exp \left(-d\left(1-\rho_{i}^{2}\right) \phi^{2}\left(t_{n}\right)\right)$, where $\sum^{(1)}$ stands for the summation over $i$ such that $\rho_{i} \geqq\left(1-\left(\log t_{n}\right)^{-1 / 2}\right)^{1 / 2}$ and $\sum^{(2)}$ stands for the remainder of $\sum$.

By the definition of $\sum^{(2)}$ and (3.2), we have, for large $n$,

$$
\left(1-\rho_{i}^{2}\right) \phi^{2}\left(t_{n}\right) \geqq\left(\log t_{n}\right)^{1 / 2}
$$

Since the number of $n_{i}$ in $\sum^{(2)}$ does not exceed $2 K\left(\log t_{n}\right)^{1 / \alpha+1}$ for large $p$,

$$
\begin{aligned}
\sum^{(2)} \exp \left(-d\left(1-\rho_{i}^{2}\right) \phi^{2}\left(t_{n}\right)\right) & \leqq \sum^{(2)} \exp \left(-d\left(\log t_{n}\right)^{1 / 2}\right) \\
& \leqq 2 K\left(\log t_{n}\right)^{1 / \alpha+1} \exp \left(-d\left(\log t_{n}\right)^{1 / 2}\right)
\end{aligned}
$$

which is bounded with respect to $p$, giving the desired result. 
Next, we shall prove that $\sum^{(1)} \exp \left(-d\left(1-\rho_{i}^{2}\right) \phi^{2}\left(t_{n}\right)\right)$ is bounded with respect to $n$. For $n_{i}$, which is added to $\sum^{(1)}$, we have

$$
1-\left(\log t_{n}\right)^{-1 / 2} \leqq \rho^{2}\left(t_{n}, t_{n^{\prime}}\right) \text {. }
$$

By assumption of Theorem 2, there is a $\delta>0$ such that if $\left|t_{n^{\prime}}-t_{n}\right|<\delta$, then $\rho\left(t_{n}, t_{n^{\prime}}\right) \leqq \eta$. Therefore, $\left|t_{n^{\prime}}-t_{n}\right|<\delta$ for large $p$. Hence, we find

$$
1-\left(\log t_{n}\right)^{-1 / 2} \leqq 1-C_{2}\left|t_{n^{\prime}}-t_{n}\right|^{\alpha},
$$

from which follows that $p^{\prime} \leqq p+1$ for large $p$. Thus, we can show that for an appropriate positive constant $D$

$$
\left(1-\rho_{i}^{2}\right) \geqq D\left(k^{\alpha} /(\log (p+1))\right) \quad \text { or } \quad D\left(k^{\alpha} /(\log p)\right),
$$

where different $i$ corresponds to different $k$. Since $\phi\left(t_{n}\right)>(\log p)^{1 / 2}$ for large $p$, we have that

$$
\sum^{(1)} \exp \left(-d\left(1-\rho_{i}^{2}\right) \phi^{2}\left(t_{n}\right)\right)<\sum_{k=0}^{\infty} \exp \left(- \text { Constant } \times k^{\alpha}\right)<+\infty,
$$

which concludes the proof of Theorem 2.

\section{REFERENCES}

1. K. L. Chung and P. Erdös, On the application of the Borel-Cantelli lemma, Trans. Amer. Math. Soc. 72 (1952), 179-186. MR 13, 567.

2. H. Cramér, On the maximum of a normal stationary stochastic process, Bull. Amer. Math. Soc. 68 (1962), 512-516. MR 25 \#3562.

3. W. Feller, The general form of the so-called law of the iterated logarithm, Trans. Amer. Math. Soc. 54 (1943), 373-402. MR 5, 125.

4. P. Lévy, Théorie de l'addition des variables aleatoires, Gauthier-Villars, Paris, 1937.

5. M. Nisio, On the extreme values of Gaussian processes, Osaka J. Math. 4 (1967), 313-326. MR 37 \#2309.

6. J. Pickands III, Maxima of stationary Gaussian processes, Z. Wahrscheinlichkeitstheorie und Verw. Gebiete 7 (1967), 190-223. MR 36 \#955.

7. M. G. Sur, On the maximum of a Gaussian process, Theor. Probability Appl. 10 (1965), 354-357.

8. T. Sirao, On the continuity of Brownian motion with a multidimensional parameter, Nagoya Math. J. 16 (1960), 135-156. MR 22 \#8570.

9. T. Sirao and T. Nisida, On some asymptotic properties concerning Brownian motion, Nagoya Math. J. 4 (1952), 97-101. MR 13, 761.

10. T. Sirao and H. Watanabe, On the upper and lower class for stationary Gaussian processes, Trans. Amer. Math. Soc. (to appear.)

11. H. Watanabe, An asymptotic property of Gaussian stationary processes, Proc. Japan Acad. 44 (1968), 895-896.

\section{KYUSHU UNIVERSITY, FUKUOKA, JAPAN}

